\title{
Kadets-Type Theorems for Partitions of a Convex Body
}

\author{
Arseniy Akopyan • Roman Karasev
}

Received: 18 November 2011 / Revised: 4 June 2012 / Accepted: 2 July 2012 /

Published online: 18 July 2012

(C) Springer Science+Business Media, LLC 2012

\begin{abstract}
For convex partitions of a convex body $B$, we try to put a homothetic copy of $B$ into each set of the partition so that the sum of the homothety coefficients is at least 1 . In the plane this can be done for an arbitrary partition, while in higher dimensions we need certain restrictions on the partition.
\end{abstract}

Keywords The Tarski plank problem · The Kadets theorem

\section{Introduction}

Alfred Tarski [12] proved that for any covering of the unit disk by planks (the sets $a \leq \lambda(x) \leq b$ for a linear function $\lambda$ and two reals $a<b$ ), the sum of plank widths is at least 2. Thøger Bang [3] generalized this result for covering of a convex body $B$ in $\mathbb{R}^{d}$ by planks showing that the sum of the widths is at least the width of $B$. He also posed the following question: Can the plank widths in the Euclidean metric be replaced by the widths relative to $B$ (as in Definition 2 below)?

\footnotetext{
A. Akopyan (凶)

Institute for Information Transmission Problems RAS, Bolshoy Karetny per. 19, Moscow, Russia 127994

e-mail: akopjan@gmail.com
}

\author{
A. Akopyan · R. Karasev \\ Dept. of Mathematics, Moscow Institute of Physics and Technology, Institutskiy per. 9, Dolgoprudny, \\ Russia 141700 \\ R. Karasev \\ e-mail: r_n_karasev@mail.ru
}

\section{A. Akopyan · R. Karasev}

Laboratory of Discrete and Computational Geometry, Yaroslavl State University, Sovetskaya st. 14, Yaroslavl, Russia 150000 
Keith Ball [2] proved the conjecture of Bang for centrally symmetric bodies $B$ or, in other words, for arbitrary normed spaces and coverings of the unit ball. For possibly nonsymmetric $B$, it is known (see [1]) that the Bang conjecture is equivalent to the Davenport conjecture: If a convex body $B$ is sliced by $n-1$ hyperplane cuts, then there exists a piece that contains a translate of $\frac{1}{n} B$.

In [5, 6] András Bezdek and Károly Bezdek proved an analogue of the Davenport conjecture for binary partitions by hyperplanes. The difference is that they do not cut everything with every hyperplane; instead, they divide one part into two parts and then proceed recursively.

One of the strongest results about coverings of a unit ball for the Hilbert (and finite-dimensional Euclidean) space was proved by Vladimir Kadets [10] (see also [4] for the proof in the two-dimensional case using the idea from [12]): For any convex covering $C_{1}, \ldots, C_{k}$ of the unit ball, the sum of the inscribed ball radii $\sum_{i=1}^{k} r\left(C_{i}\right)$ is at least 1.

The reader is referred to [7] for a detailed historical survey on the Tarski plank problem. Some other open problems can be found in [9].

In this paper we prove analogues of the Kadets theorem for inscribing homothetic copies of a (not necessarily symmetric) convex body, replacing arbitrary coverings by certain convex partitions. By a partition of a convex set $B$ we mean a covering of $B$ by a family of closed convex sets with disjoint interiors. In the two-dimensional case the analogue of the Kadets theorem for possibly nonsymmetric bodies (Theorem 2) holds for any partition, while in higher dimensions we need additional restrictions on the partition. In other words, we are solving positively certain particular cases of [7, Problem 4.4.2] about extending the Kadets theorem to Banach spaces.

We work in finite-dimensional spaces. If one needs analogues for infinitedimensional Banach spaces, then the standard approximation argument works as in [10].

This paper is organized as follows. In Sect. 2 we define inductive partitions and prove a Kadets-type result for such partitions. In Sect. 3 we introduce affine partitions and hierarchical affine partitions, which are very natural examples of inductive partitions.

The main result of the paper, the case of two-dimensional partition, is proved in Sect. 4. In Sect. 5 we introduce inductive coverings. This is a special type of coverings, for which our methods prove a Kadets-type theorem. Section 6 is not connected with the previous sections directly; there we give a simplified proof of the BezdekSchneider analogue of the Kadets theorem on the sphere. In Sect. 7 we show that in the hyperbolic case the Kadets theorem does not hold.

\section{Inductive Partitions}

Let us describe the class of partitions, for which an analogue of the Kadets theorem is true:

Definition 1 A convex partition $V_{1} \cup \cdots \cup V_{k}$ of $\mathbb{R}^{d}$ is said to be inductive if for any $1 \leq i \leq k$, there exists an inductive partition $W_{1} \cup \cdots \cup W_{i-1} \cup W_{i+1} \cup \cdots \cup W_{k}$ 
Fig. 1 Not inductive partition

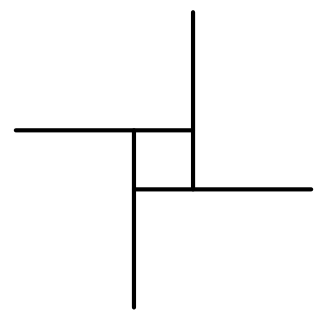

such that $W_{j} \supseteq V_{j}$ for any $j \neq i$. A partition into one part $V_{1}=\mathbb{R}^{d}$ is assumed to be inductive.

A natural example of an inductive partition is a Voronoi partition or, more generally, a projection of lower faces of a convex polytope. In the next section we generalize these examples and discuss hierarchical affine partitions, which are the only examples of inductive partitions that we know. An example of a noninductive partition is outlined on Fig. 1.

Now we define the inradius relative to $B$ :

Definition 2 Let $B \subset \mathbb{R}^{d}$ be a convex body. For a convex set $C \subseteq \mathbb{R}^{d}$, define the analogue of the inscribed ball radius as follows:

$$
r_{B}(C)=\sup \left\{h \geq 0: \exists t \in \mathbb{R}^{d} \text { such that } h B+t \subseteq C\right\}
$$

and $r_{B}(C)=-\infty$ for empty $C$.

Now we are ready to state one of the main results.

Theorem 1 Let $B \subset \mathbb{R}^{d}$ be a convex body, and let $C_{1} \cup \cdots \cup C_{k}=B$ be induced from an inductive partition $V_{1} \cup \cdots \cup V_{k}=\mathbb{R}^{d}$ (that is, $C_{i}=V_{i} \cap B$ for any $i$ ). Then

$$
\sum_{i=1}^{k} r_{B}\left(C_{i}\right) \geq 1 .
$$

Before proving this theorem we need a lemma about the inradius.

Lemma 1 Let a convex polytope $C \subset \mathbb{R}^{d}$ be defined by linear inequalities for $i=$ $1, \ldots, m$ :

$$
\lambda_{i}(x) \leq 0 .
$$

Denote by $C(\bar{y})$ the polytope defined by the inequalities

$$
\lambda_{i}(x)+y_{i} \leq 0
$$

where $\bar{y}=\left(y_{1}, \ldots, y_{m}\right)$ is a vector of reals. Then $r_{B}(C(\bar{y}))$ is a concave function of $\bar{y}$. 
Proof Denote the set of indices $[m]=\{1, \ldots, m\}$. By the Helly theorem we have

$$
r_{B}(C(\bar{y}))=\inf _{I \subseteq[m],|I| \leq d+1} r_{B}\left(C_{I}(\bar{y})\right),
$$

where $C_{I}(\bar{y})$ is defined by the inequalities $\lambda_{i}(x)+y_{i} \leq 0$ for $i \in I$. The sets $C_{I}(\bar{y})$ are either Cartesian products of a linear subspace $L \subset \mathbb{R}^{d}$ of positive dimension with a lower-dimensional polyhedral set $C_{I}^{\prime}(\bar{y})$, or simplicial cones, or simplices. In the first case we use induction on the dimension. In the second case we note that $r_{B}\left(C_{I}(\bar{y})\right)=$ $+\infty$. In the third case the function $r_{B}\left(C_{I}(\bar{y})\right)$ is obviously linear. Hence, for any $C_{I}(\bar{y})$, the inradius $r_{B}\left(C_{I}(\bar{y})\right)$ is a concave function of $\bar{y}$. Therefore the inradius $r_{B}(C(\bar{y}))$ is concave as an infimum of concave functions.

Lemma 2 Let $C_{1}, \ldots, C_{m}$ be a family of convex bodies in $\mathbb{R}^{d}$. Then the inradius of the intersection of translates

$$
r_{B}\left(\left(C_{1}+y_{1}\right) \cap\left(C_{2}+y_{2}\right) \cap \cdots \cap\left(C_{m}+y_{m}\right)\right)
$$

is a concave function of $\bar{y}=\left(y_{1}, \ldots, y_{m}\right) \in\left(\mathbb{R}^{d}\right)^{\times m}$.

Proof When $C_{i}$ 's are polytopes, this is a particular case of Lemma 1. The general case is made by approximating $C_{i}$ 's by polytopes and passing to the limit.

Remark 1 In the above lemmas we actually prove that the set of vectors $\bar{y}$ such that the considered function of $\bar{y}$ is $>-\infty$ is a convex closed set.

Proof of Theorem 1 Let us vary the vector $y \in \mathbb{R}^{d}$ and define $C_{i}(y)=B \cap\left(V_{i}+y\right)$. The function $r(y)=\sum_{i=1}^{k} r_{B}\left(C_{i}(y)\right)$ is a concave function of $y$ by Lemma 2 , and the set $Y=\{y: r(y)>-\infty\}$ is a convex closed set. If $y$ is in the boundary of $Y$, then at least one of $C_{i}(y)$ has the empty interior. In this case we can omit the corresponding $V_{i}$ and consider a smaller partition $\left\{W_{j}\right\}_{j \neq i}$, which induces the same partition $\left\{\left(W_{j}+\right.\right.$ $y) \cap B\}_{j \neq i}$ as $\left\{\left(V_{j}+y\right) \cap B\right\}$ up to sets with empty interior.

Thus, by induction we have $r(y) \geq 1$ on $\partial Y$. Along with the concavity of $r(y)$, this implies $r(y) \geq 1$ on the whole $Y$ unless $Y$ is a halfspace. From the obvious formula (the sum is the Minkowski sum)

$$
Y=\bigcap_{i=1}^{k}\left(B+\left(-V_{i}\right)\right)
$$

it follows that $Y$ can be a halfspace if and only if every $V_{i}$ contains the same halfspace. This is impossible unless $k=1$; but for $k=1$, the theorem is obviously true.

\section{Affine Partitions}

In this section we describe constructively a certain class of inductive partitions. 
Definition 3 For a sequence of affine (linear with possible constant term) functions $F=\left\{\lambda_{1}, \ldots, \lambda_{k}\right\}$, define the affine partition $P(F)$ of $\mathbb{R}^{d}$ by

$$
C_{i}=\left\{x \in \mathbb{R}^{d}: \forall j \neq i, \text { we have } \lambda_{i}(x) \leq \lambda_{j}(x)\right\} .
$$

An affine partition of a subset $X \subset \mathbb{R}^{d}$ is defined as a restriction of an affine partition of the whole $\mathbb{R}^{d}$.

Remark 2 Affine partitions are also known as generalized Voronoi partitions or power diagrams, but we use the term affine for brevity.

Corollary 1 Let $B \subset \mathbb{R}^{d}$ be a convex body, and let $C_{1} \cup \cdots \cup C_{k}=B$ be its affine partition. Then

$$
\sum_{i=1}^{k} r_{B}\left(C_{i}\right) \geq 1 .
$$

Proof It suffices to show that any affine partition is inductive. Starting from $V_{1} \cup$ $\cdots \cup V_{k}=\mathbb{R}^{d}$ defined by $\left\{\lambda_{1}, \ldots, \lambda_{k}\right\}$, we omit $\lambda_{i}$ from the list and obtain another affine partition $\left\{W_{j}\right\}_{j \neq i}$ such that $W_{j} \supseteq V_{j}$ for any $j \neq i$. So the induction step is possible.

A straightforward generalization of an affine partition is a hierarchical affine partition:

Definition 4 By induction: If in a hierarchical affine partition $C_{1} \cup \cdots \cup C_{k}$ we partition some $C_{i}$ by an affine partition, we obtain again a hierarchical affine partition.

Let us show that a hierarchical affine partition is a limit of affine partitions.

Lemma 3 Suppose that $B$ is a convex body and $C_{1} \cup \cdots \cup C_{k}=B$ is its hierarchical affine partition. Then this partition can be approximated by an affine partition with arbitrary precision in the Hausdorff metric.

Proof From the definition we know that there exists a graded tree $T$ with an affine function $\lambda_{v}$ in every vertex $v \in T$ such that the sets $C_{i}$ correspond to the leaves $\ell_{i}$ of $T$; the condition $x \in C_{i}$ is equivalent to $\lambda_{v}(x) \leq \lambda_{w}(x)$ for any $v$ in the ancestors of $\ell_{i}$ and $w$ a sibling of $v$.

Now we take $\varepsilon>0$ small enough and for any $C_{i}$ and its corresponding $\ell_{i}$, consider the full chain from the root $v_{0}<v_{1}<\cdots<v_{m}=\ell_{i}$ and the corresponding affine function

$$
\lambda_{i, \varepsilon}=\lambda_{v_{0}}+\varepsilon \lambda_{v_{1}}+\cdots+\varepsilon^{m} \lambda_{v_{m}} .
$$

Now it is obvious that the affine partition of $B$ corresponding to $\left\{\lambda_{i, \varepsilon}\right\}_{i=1}^{k}$ tends to $\left\{C_{i}\right\}_{i=1}^{k}$ as $\varepsilon$ tends to +0 . 
Even without this lemma it is obvious that Corollary 1 holds for hierarchical affine partitions by induction. Note that a binary partition by hyperplanes is a particular case of a hierarchical affine partition.

\section{The Two-Dimensional Case}

Now we are ready to prove an analogue of the Kadets theorem in the plane. The key property of an inductive partition in the proof of Theorem 1 is actually the following: we consider convex partitions $C_{1} \cup \cdots \cup C_{k}=B$ that can be extended to a convex partition $V_{1} \cup \cdots \cup V_{k}=\mathbb{R}^{d}$. Then we can translate $V_{i}$ 's with $y$ so that one of the sets $C_{i}=V_{i} \cap B$ disappears, remove $C_{i}$, extend the partition $\left\{C_{j}\right\}_{j \neq i}$ again to a new partition of the whole space, and so on.

In the plane the extension is always possible by the following:

Lemma 4 Any convex partition $C_{1} \cup \cdots \cup C_{k}=B \subset \mathbb{R}^{2}$ can be extended to a partition $V_{1} \cup \cdots \cup V_{k}=\mathbb{R}^{2}$.

Proof The boundary $\partial B$ consists of parts of the boundaries $\partial C_{i}$. Denote the vertices of this partition by $a_{1}, a_{2}, \ldots, a_{n}$. Denote the polygon $a_{1} a_{2} \cdots a_{n}$ by $A$. Note that for some $C_{i}$ s, we may have more than one corresponding part of $\partial B$.

Obviously, from each point $a_{i}$ it is possible to draw a ray $\ell_{i}$ outside $B$ with the following property: For any set $C_{j}$ and its corresponding boundary segment $\left[a_{i} a_{i+1}\right]$ (the indices are understood cyclically $a_{n+1}=a_{1}$ ), the union of $C_{j}$ and the area that is bounded by $\left[a_{i} a_{i+1}\right], \ell_{i}$, and $\ell_{i+1}$ is convex. For the rays $\ell_{i}$, one can take the extension of the interior with respect to $B$ side of $C_{j}$ after $a_{i}$.

Our goal is to erase parts of the rays $\ell_{i}$ and obtain a partition of $\mathbb{R}^{2} \backslash A$ into $n$ convex parts. At the start the rays may partition $\mathbb{R}^{2} \backslash A$ into a larger number of parts.

We perform erasing as follows. Suppose that $b_{j}$ is a point of transversal intersection of two rays $\ell_{s}$ and $\ell_{t}$ that is closer to $A$ than other points of transversal intersection of the remaining rays. Note that the segments $b_{j} a_{s}$ and $b_{j} a_{t}$ do not intersect with other rays $\ell_{i}$ transversally (in this case the point of intersection would be closer to $A$ than $b_{j}$ ). Erase part of one of the rays $\ell_{s}$ or $\ell_{t}$ after $b_{i}$ and start a new iteration of this process again. Some rays actually become segments, but it does not matter.

After each step we have a convex partition of $\mathbb{R}^{2} \backslash A$, and finally we obtain a partition into exactly $n$ parts.

After taking union of these parts with their corresponding sets $C_{i}$, we obtain the required extension of the partition to the whole plane, see Fig. 2.

Now we are ready to state the result.

Theorem 2 Let $B \subset \mathbb{R}^{2}$ be a convex body, and let $C_{1} \cup \cdots \cup C_{k}=B$ be its convex partition. Then

$$
\sum_{i=1}^{k} r_{B}\left(C_{i}\right) \geq 1
$$


Fig. 2 Extending the partition

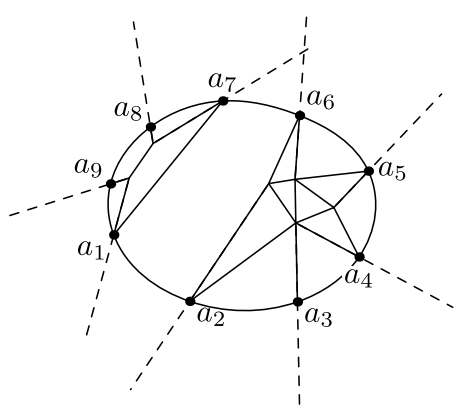

Proof We extend the partition $C_{1} \cup \cdots \cup C_{k}=B$ to $V_{1} \cup \cdots \cup V_{k}=\mathbb{R}^{2}$ by Lemma 4 . Then the function

$$
r(y)=\sum_{i=1}^{k} r_{B}\left(B \cap\left(V_{i}+y\right)\right)
$$

is again concave, so by varying $y$ we can make one of $B \cap\left(V_{i}+y\right)$ have the empty interior without increasing $r(y)$. Then we omit $V_{i}$, obtain a partition of $B$ into fewer parts, and use the inductive assumption.

\section{Possible Extension to Coverings}

Theorem 2 is quite close to the plane case of the Bang conjecture, which we restate here: If $B \subset \mathbb{R}^{2}$ is covered by a set of planks $W_{1} \cup \cdots \cup W_{k} \supseteq B$, then $\sum_{i=1}^{k} r_{B}\left(W_{i}\right) \geq 1$. The key difference is that in the Bang conjecture we have a covering, not a partition. Intuitively, a partition is something smaller than covering and therefore has smaller sum of "inradii." But already in the case of $\mathbb{R}^{2}$ there exist coverings that do not contain partitions. A simple example is a set of planks $C_{i}$ passing through the center of a disk $B$, forming a "sunflower" so that each of the sets $C_{i} \cap \partial B$ consists of two disjoint arcs and these arcs partition $\partial B$.

It is easily verified that Theorem 1 holds (with the same proof literally) for coverings instead of partitions if we define an inductive covering by the following:

Definition 5 Call a convex covering (by closed sets) $V_{1} \cup \cdots \cup V_{k}$ of $\mathbb{R}^{d}$ inductive if for any $1 \leq i \leq k$, there exists an inductive covering $W_{1} \cup \cdots \cup W_{i-1} \cup W_{i+1} \cup \cdots \cup W_{k}$ such that $W_{j} \subseteq V_{j} \cup V_{i}$ for any $j \neq i$. A covering by one set $V_{1}=\mathbb{R}^{d}$ is assumed to be inductive.

Returning to the Bang conjecture, we see the unpleasant thing: If we cover some part of $\mathbb{R}^{2}$ by planks and the remaining part is covered by the corresponding (possibly infinite) polygons, then none of the polygons can be deleted, so this is an example of a noninductive covering of the plane. 


\section{Notes on the Spherical Kadets Theorem}

In [8] Károly Bezdek and Rolf Schneider proved the following version of the Kadets theorem in the spherical geometry:

Theorem 3 (K. Bezdek, R. Schneider, 2010) If the sphere $\mathbb{S}^{n}$ is covered by spherical convex sets $K_{i}$, then we have the following inequality for the inradii:

$$
\sum_{i} r\left(K_{i}\right) \geq \pi
$$

Here we use the standard intrinsic metric of the unit sphere. By a convex set we mean the intersection of this sphere and a convex cone whose apex is at the center of the sphere.

This theorem gives rise to the following:

Problem 1 (K. Bezdek, R. Schneider, 2010) Suppose that $B_{\rho} \subset \mathbb{S}^{n}$ is a ball of radius $\rho$ in the spherical geometry. Suppose that $B_{\rho}$ is covered by spherical convex sets $K_{i}$. Prove that

$$
\sum_{i} r\left(K_{i}\right) \geq \rho
$$

As it is noted in [8], Theorem 3 solves this problem for $\rho \geq \pi / 2$, the solution being essentially volumetric. But if $\rho \rightarrow 0$, then this problem approaches the original Kadets theorem, which has no volumetric solution for $n>2$. So it seems that solving this problem for $\rho$ in the range $(0, \pi / 2)$ must require a new approach.

Let us outline the proof of Theorem 3. This proof is essentially the same as that given in [8]; but we simplify it and split into several lemmas, some of which may be of interest on their own.

Lemma 5 Let $\mu$ be a spherically symmetric absolute continuous measure on $\mathbb{R}^{n}, B$ be a ball centered at the origin, and $T$ be a 0 -starshaped body. Then

$$
\mu(B \cap T) \mu\left(\mathbb{R}^{n}\right) \geq \mu(B) \mu(T) .
$$

Proof The proof will use a very simple case of the needle decomposition (see [11] for example). Let us split $\mathbb{R}^{n}$ into convex cones $V_{i}$ of equal measures $\mu\left(V_{i}\right)$. Note that the sets $V_{i} \cap B$ will also have equal measures because of the spherical symmetry of $\mu$. The lemma will follow from the inequality

$$
\mu\left(B \cap T \cap V_{i}\right) \mu\left(V_{i}\right) \geq \mu\left(B \cap V_{i}\right) \mu\left(T \cap V_{i}\right)
$$

by summation. The partition can be made so that every $V_{i}$ gets arbitrarily close to a one-dimensional ray, and the limit case of (1) becomes an inequality for nonnegative functions:

$$
\int_{0}^{\min \{x, y\}} f(t) d t \cdot \int_{0}^{+\infty} f(t) d t \geq \int_{0}^{x} f(t) d t \cdot \int_{0}^{y} f(t) d t,
$$


which simply follows from the observation that $\min \{X, Y\} Z \geq X Y$ for any $X, Y \in$ $[0, Z]$.

Lemma 6 Let us work in the spherical geometry. Suppose that $H \subset \mathbb{S}^{n}$ is a hemisphere with center $o, B$ is a ball of radius $\leq \pi / 2$ centered at $o$, and $T$ is an $o$ starshaped body in $H$. Then

$$
\sigma(B \cap T) \sigma(H) \geq \sigma(B) \sigma(T)
$$

for the standard measure $\sigma$ on the sphere.

Proof Follows from Lemma 5 by central projection of $H$ onto $\mathbb{R}^{n}$ such that $o$ goes to 0 .

Lemma 7 Let $X$ be s subset of the sphere $\mathbb{S}^{n}$ not contained in an open hemisphere, and $X_{0}$ be a set consisting of two antipodal points on the sphere. Then for their $\varepsilon$ neighborhoods (in the spherical geometry), we have

$$
\sigma(X+\varepsilon) \geq \sigma\left(X_{0}+\varepsilon\right)
$$

Proof Without loss of generality, let $X=\left\{o_{1}, \ldots, o_{m}\right\}$ be finite. Consider the hemispheres $H_{i}$ with respective centers $o_{i}$ and the Voronoi regions $V_{i}$ of $o_{i}$. Note that $V_{i} \subseteq H_{i}$ for every $i$. Denote the measure of the whole sphere $\mathbb{S}^{n}$ by $\sigma_{n}$.

Then by Lemma 6

$$
\sigma\left(V_{i} \cap B_{o_{i}}(\varepsilon)\right) \frac{\sigma_{n}}{2} \geq \sigma\left(B_{o_{i}}(\varepsilon)\right) \sigma\left(V_{i}\right)
$$

hence,

$$
\sigma\left(V_{i} \cap(X+\varepsilon)\right) \frac{\sigma_{n}}{2} \geq \sigma\left(B_{o_{i}}(\varepsilon)\right) \sigma\left(V_{i}\right),
$$

and then by summing over $i$ and multiplying by 2 ,

$$
\sigma(X+\varepsilon) \sigma_{n} \geq 2 \sigma\left(B_{*}(\varepsilon)\right) \sigma_{n},
$$

where $B_{*}(\varepsilon)$ is any ball (on the sphere) of radius $\varepsilon$. So we obtain

$$
\sigma(X+\varepsilon) \geq 2 \sigma\left(B_{*}(\varepsilon)\right)=\sigma\left(X_{0}+\varepsilon\right) .
$$

Lemma 8 Let $\mu$ be an absolutely continuous spherically symmetric measure on $\mathbb{R}^{n}$. Suppose that $K$ is a convex body in $\mathbb{R}^{n}$ with inscribed ball $B$, centered at the origin. Then

$$
\mu(K) \leq \mu(T),
$$

where $T$ is a plank with inscribed ball $B$. 
Proof Representing the measure $\mu$ as an integral, it suffices to prove the inequality

$$
\sigma(K \cap S) \leq \sigma(T \cap S)
$$

for any sphere $S$ centered at the origin. Let the radii of $B$ and $S$ be $r$ and $R$, respectively. For $R \leq r$, inequality (2) is obvious, so we consider $R>r$.

The set $T \cap S$ is the complement of the $\varepsilon$-neighborhood of two opposite points $X_{0}$ in $S$, where $\varepsilon=\arccos r / R$. Since $K$ has $B$ as the inscribed ball, the set $X^{\prime}=\partial K \cap B$ contains the origin in its convex hull. It is easy to see that the set $X=R / r X^{\prime}$ is not contained in an open hemisphere and that its $\varepsilon$-neighborhood is disjoint with $K \cap S$. By Lemma 7,

$$
\sigma(X+\varepsilon) \geq \sigma\left(X_{0}+\varepsilon\right)
$$

hence

$$
\sigma(K \cap S) \leq \sigma(S \backslash(X+\varepsilon)) \leq \sigma\left(S \backslash\left(X_{0}+\varepsilon\right)\right)=\sigma(T \cap S),
$$

which is exactly (2).

Now we deduce the following (the same as [8, Theorem 2]):

Lemma 9 Suppose that $K$ is a convex body in $\mathbb{S}^{n}$ with inscribed ball B. Then

$$
\sigma(K) \leq \sigma\left(K_{0}\right)
$$

where $K_{0}=H_{0} \cap H_{1}$ is an intersection of two hemispheres with inscribed ball $B$. Note that $\sigma\left(K_{0}\right)$ equals $\frac{\alpha \sigma_{n}}{2 \pi}$, where $\alpha$ is the angle between $H_{0}$ and $H_{1}$.

Proof Obtained from Lemma 8 by central projection that takes the center of $B$ to the origin in $\mathbb{R}^{n}$.

Now Theorem 3 follows from Lemma 9 by bounding from above the volume of every $K_{i}$ in terms of $r\left(K_{i}\right)$.

\section{The Hyperbolic Kadets Theorem}

It is interesting that the Kadets theorem does not hold for hyperbolic space unlike the spherical case mentioned above. We skip the calculation here because of the negativity of this result, but the figures should be sufficiently convincing.

Consider a sufficiently large disk $\Omega$ and a regular hexagon inscribed in it. Let us cover this disk by two convex shapes, which are drawn in Fig. 3 (this is the Poincaré model). The maximal inscribed disk of a shape is drawn by a dashed line. Since it does not contain the center of $\Omega$, its radius is less than half of the radius of $\Omega$.

Note that this counterexample uses essentially that $C_{1}$ and $C_{2}$ do intersect. The authors do not know whether the Kadets theorem holds for partitions in the hyperbolic space. 
Fig. 3 Disk covering


Acknowledgements The research of both authors is supported by the Dynasty Foundation, the President's of Russian Federation grant MD-352.2012.1, the Russian Foundation for Basic Research grant 1001-00096, the Federal Program "Scientific and scientific-pedagogical staff of innovative Russia" 20092013, and the Russian government project 11.G34.31.0053. The research of A.V. Akopyan is also supported by the Russian Foundation for Basic Research grant 11-01-00735, and the research of R.N. Karasev is also supported by the Russian Foundation for Basic Research grant 10-01-00139.

\section{References}

1. Alexander, R.: A problem about lines and ovals. Am. Math. Mon. 75, 482-487 (1968)

2. Ball, K.: The plank problem for symmetric bodies. Invent. Math. 104(3), 535-543 (1991). doi:10. 1007/BF01245089

3. Bang, T.: A solution of the "plank problem". Proc. Am. Math. Soc. 2, 990-993 (1951)

4. Bezdek, A.: On a generalization of Tarski's plank problem. Discrete Comput. Geom. 38(2), 189-200 (2007). doi:10.1007/s00454-007-1333-8

5. Bezdek, A., Bezdek, K.: A solution of Conway's fried potato problem. Bull. Lond. Math. Soc. 27(5), 492 (1995)

6. Bezdek, A., Bezdek, K.: Conway’s fried potato problem revisited. Arch. Math. 66(6), 522-528 (1996)

7. Bezdek, K.: Classical Topics in Discrete Geometry. Springer, Berlin (2010). doi:10.1007/978-14419-0600-7

8. Bezdek, K., Schneider, R.: Covering large balls with convex sets in spherical space. Contrib. Algebra Geom. 51(1), 229-235 (2010)

9. Brass, P., Moser, W., Pach, J.: Research Problems in Discrete Geometry. Springer, New York (2005)

10. Kadets, V.: Coverings by convex bodies and inscribed balls. Proc. Am. Math. Soc. 133(5), 1491-1496 (2005)

11. Nazarov, F., Sodin, M., Vol'berg, A.: The geometric Kannan-Lovász-Simonovits lemma, dimensionfree estimates for the distribution of the values of polynomials, and the distribution of the zeros of random analytic functions. St. Petersburg Math. J. 14(2), 351-366 (2002)

12. Tarski, A.: Further remarks about the degree of equivalence of polygons. Odbitka Z. Parametr. 2, 310-314 (1932) 